\title{
Determination of the Dill parameters of thick positive resist for use in modeling applications
}

\author{
G. Roeder ${ }^{\mathrm{a}, *}$, S. Liu ${ }^{\mathrm{a}}$, G. Aygun ${ }^{\mathrm{a}, \mathrm{b}}$, P. Evanschitzky ${ }^{\mathrm{a}}$, A. Erdmann ${ }^{\mathrm{a}, \mathrm{c}}$, M. Schellenberger ${ }^{\mathrm{a}}$, L. Pfitzner ${ }^{\mathrm{a}}$ \\ ${ }^{a}$ Fraunhofer Institute for Integrated Systems and Device Technology (IISB), Schottkystrasse 10, 91058, Erlangen, Germany \\ b Physics Department, Izmir Institute of Technology, 35430, Urla, Izmir, Turkey \\ c Erlangen Graduate School in Advanced Optical Technologies (SAOT), 91058, Erlangen, Germany
}

\section{A R T I C L E I N F O}

Available online 20 December 2010

\section{Keywords:}

Spectroscopic ellipsometry

Dill parameters

Thick resist

Simulation

\begin{abstract}
A B S T R A C T
The determination of Dill parameters of thick resist is very important to improve simulation models of resist exposure and real world processes. A new extraction technique of Dill parameters based on spectroscopic ellipsometry in combination with an advanced resist exposure model is proposed for thick resist analysis. The complex refractive index of the resist is related to the relative concentration of the photoactive compound in the resist in order to describe the vertical distribution of the refractive index and the extinction coefficient. Moreover, Dill parameters are extracted by directly fitting the bleaching curves to the measured ellipsometry data. The new approach was investigated experimentally by spectroscopic ellipsometry measurements on AZ5214E resist with two moderate layer thickness values in order to verify the accuracy of the new method. Dill parameters were extracted by using this new technique and by applying resist samples subjected to different exposure doses. Possible reasons for the variation of Dill parameters depending on resist thickness are explained. Furthermore, advantages, limitations and potential improvements of the model are discussed. Finally, the impact of Dill parameter variation on image formation in the resist is demonstrated by applying the spectroscopic ellipsometer analysis results as input parameters to the lithography simulator Dr.LiTHO.
\end{abstract}

(C) 2011 Elsevier B.V. All rights reserved.

\section{Introduction}

Thick film photoresists with thickness from several micrometers up to several hundred micrometers are extensively used in the fabrication of thin film heads, solder bonding, and micro-electromechanical systems due to their excellent performance and low processing cost [1]. Thick photoresists are still continuing to gain importance for advanced chip scale packing and microsystems including biomedical sensors, optical switches, and accelerometers. Similar to the situation in semiconductor manufacturing, the application of optical lithography simulation can help to optimize the complex lithography processes, increase the understanding of new processes and reduce development costs. For thicknesses in the range of $1 \mu \mathrm{m}$, a variety of resist types were studied in the past to determine relevant input parameters for simulation tools. However, simulation tools for thin film lithography have to be further developed to be able to consider the nonlinear properties of thick photoresists by improving the simulation models such as the Dill exposure model [2,3] and the Mack development model [4,5]. For model improvement, accurate measurement of the simulation parameters for thick photoresist is an important issue.

\footnotetext{
* Corresponding author.

E-mail address: georg.roeder@iisb.fraunhofer.de (G. Roeder).
}

To overcome the limitation of transparent substrate in the traditional extraction techniques of exposure parameters (so called Dill parameters) [6], Schiavone et al. [7] proposed a method for the determination of Dill parameters based on reflectivity measurement as a function of exposure dose. Even though the method is simple and is applicable to any kind of substrate, it is very sensitive to the determination of the refractive index and thickness of resist. To accurately obtain both the refractive index $n$ and the extinction coefficient $k$ of resist at different exposure doses, Boher et al. [8] and Henderson et al. [9] employed spectroscopic ellipsometry (SE) to measure $\tan (\Psi)$ and $\cos (\Delta)$ of reflected light instead of photometry to measure the reflected intensity from the surface of resist. In [8] an in situ set-up and a step-by-step exposure procedure to determine the Dill parameters are reported. A single-film model with constant $n$ and $k$ values was used to simulate the resist for each exposure dose. With this single-film model, important effects during resist exposure such as the formation of standing waves and the decomposition of the photoactive compound (PAC) which lead to non-uniform film properties vs. resist thickness cannot be considered. These nonuniformity effects are present already at low resist thickness of about $1 \mu \mathrm{m}$ and will be more pronounced at larger resist thicknesses. In this paper, a new extraction technique of Dill parameters for thick resist based on SE is proposed where these effects are considered by applying a sophisticated lithography simulator to calculate the optical film model. In this optical model, the refractive index of the resist is 
related to the relative concentration of the photoactive compound in the resist in order to describe the vertical distribution of $n$ and $k$ values. Moreover, the bleaching curves of $\tan (\Psi)$ and $\cos (\Delta)$ instead of the absorption coefficient are directly fitted to extract the Dill parameters. Measurements on resist subjected to different exposure doses by SE were performed experimentally to characterize the resist and to verify the accuracy of the new method. Dill parameters of resists with two thicknesses were extracted by using this new technique. The variations of Dill parameters with respect to thickness are explained. Finally, the impact of Dill parameters variation on image formation in the resist is demonstrated by using the lithography software Dr.LiTHO [10].

\section{New extraction technique of Dill parameters based on spectroscopic ellipsometry}

\subsection{Dill exposure model and refractive index distribution in the resist}

The Dill exposure model is a classical model to describe the exposure process of photoresists by using the internal intensity distribution and PAC concentration coupled in two differential equations. By using a quasi-static approximation, the relative PAC concentration at time $t$ can be written as,

$m(z, t)=m\left(z, t_{0}\right) \exp [-C \cdot D]$

where $m\left(z, t_{0}\right)$ is the relative PAC concentration at initial time, $t_{0}\left(t_{0}=0, m\left(z, t_{0}\right)=1\right)$. $C$ is the standard exposure rate constant that describes the efficiency of the photochemical reaction, and $D$ is the exposure dose during a time interval $\Delta t$,

$D=I(z, t) \cdot \Delta t$

where $I(z, t)$ is the intensity distribution at time $t$ in the resist.

Once the PAC concentration is updated during an incremental dose step, the refractive index of the resist will be changed. This process is referred to as the bleaching effect. A linear relationship between the refractive index $n$ and the local relative PAC concentration is used to describe the bleaching effect as,

$n(z, t)=n_{\text {bleached }}+m(z, t)\left(n_{\text {unbleached }}-n_{\text {bleached }}\right)$

where $n_{\text {unbleached }}$ and $n_{\text {bleached }}$ are the refractive index of the resist before the exposure process and after being fully exposed. In addition, the extinction coefficient $k$ can also be obtained by the relative PAC concentration as,

$k(z, t)=\lambda[A \cdot m(z, t)+B] / 4 \pi$

where $A$ and $B$ are the other two Dill parameters referred, respectively, as the bleachable absorbance and the non-bleachable absorbance of the photoresist, and $\lambda$ is the exposure wavelength.

Eqs. (1)-(4) describe the exposure process of resist with monochromatic light. In the mask aligner investigated here, broadband illumination by a Hg-lamp is applied, which includes three main wavelengths, i.e. $365 \mathrm{~nm}, 405 \mathrm{~nm}$, and $436 \mathrm{~nm}$. In this case, the three lines may contribute simultaneously to the PAC decomposition depending on the photoresist sensitivity. The relative changes of the PAC concentration induced by each individual line are responsible for the total bleaching of the resist. Assuming the initial relative PAC concentration value as 1 , the resulting relative change of $m(z, t)$ PAC concentration can be written as,

$m(z, t)=e^{-\left(C_{365 n m} \cdot D_{365 n m}+C_{405 n m} \cdot D_{405 n m}+C_{436 n m} \cdot D_{436 n m}\right)}$

where $C_{365 \mathrm{~nm}}, C_{405 \mathrm{~nm}}, C_{436 \mathrm{~nm}}$ are the standard exposure rate constants, and $D_{365 \mathrm{~nm}}, D_{405 \mathrm{~nm}}$ and $D_{436 \mathrm{~nm}}$ are the doses within the resist at the three different exposure wavelengths. Then, the refractive index of $n$ and $k$ at each exposure wavelength can be obtained by substituting Eq. (5), respectively, into Eq. (3) and Eq. (4). However, the contribution of the PAC decomposition by the individual exposure wavelengths is not accessible, e.g. from the measured $n, k$ values with respect to dose values. For broadband illumination, the experimentally obtained $n, k$ values at a single measurement wavelength are rather described by an effective $C_{\text {eff }}$ value at each exposure wavelength. Then, the respective PAC decomposition effect on the other exposure wavelengths is taken into account with a normalized exposure dose deposited in the resist at the respective wavelength. Rearranging Eq. (5) yields

$m(z, t)=e^{\left(-D_{365 n m}\left(C_{365 n m}+C_{405 n m} \cdot \frac{D_{405 n m}}{D_{365 n m}}+C_{436 n n} \cdot \frac{D_{436 n m}}{D_{365 n m}}\right)\right)}=e^{-C_{365 n m, e f f} \cdot D_{365 n m}}$

with $C_{365 n m, e f f}$ as an effective parameter. Similarly, the effective Dill parameter $C_{\text {eff }}$ can be respectively defined at $405 \mathrm{~nm}$ and $436 \mathrm{~nm}$. The effective values can be used to describe the broadband illumination exposure process using a reference wavelength and including the contributions of other wavelengths on the PAC decomposition. Here, all relevant optical parameters of the exposure setup, e.g. the intensities of the different wavelengths, and the $n, k$ values of the resist at the different wavelengths have to be known.

An exposure process of AZ5214E resist coated on silicon wafer with $405 \mathrm{~nm}$ ultraviolet (UV) light is simulated. As main results of the simulation process, the light intensity distribution induced by standing waves, the PAC concentration distribution and the distribution of the refractive index as well as the extinction coefficient in the resist according to Eqs. (1) and (5) are obtained. The simulation result of the vertical refractive index distribution in the resist with respect to the depth into the resist is shown in Fig. 1. The effective refractive index based on a single-film model is also given. It is seen that the refractive index of the resist shows a graded distribution due to absorption of light and standing wave distribution of the PAC concentration inside the resist. Therefore, a single-film model with a constant refractive index, especially for thick film resist, is not adequate for the extraction of resist properties, e.g. the Dill parameters at the exposure wavelengths. However, the relative PAC concentrations of the unexposed and fully exposed resist are 1 and 0 , respectively. Here, the resist can be treated with a single-layer model for extraction of the $n, k$ values of unexposed and fully exposed resist from the SE measurements, e.g. by a point-by-point fit. Additionally, advantages of a single-layer model can be taken into account to determine the thickness of the resist where it is applicable.

To quantify the difference of SE signals between the single-film model and the new model, we calculate the SE signals, i.e. $\tan (\Psi)$ and

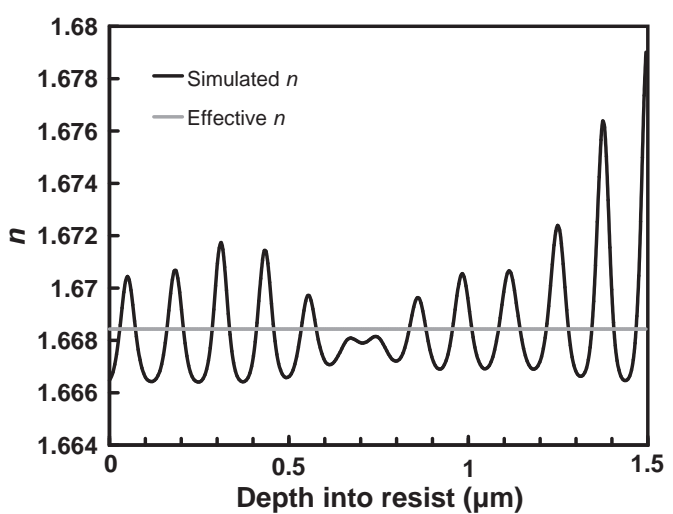

Fig. 1. Simulation result of the refractive index distribution with respect to depth for $1.5 \mu \mathrm{m}$ thick resist at $\lambda=405 \mathrm{~nm}$. 
$\cos (\Delta)$ by using the transfer matrix method. An error function $\xi$ is used in the evaluation process,

$\xi=\frac{1}{2}\left(\left|\frac{\tan \left(\Psi_{0}\right)-\tan \left(\Psi_{1}\right)}{\tan \left(\Psi_{0}\right)}\right|+\left|\frac{\cos \left(\Delta_{0}\right)-\cos \left(\Delta_{1}\right)}{\cos \left(\Delta_{0}\right)}\right|\right)$

where $\tan \left(\Psi_{0}\right)$ and $\cos \left(\Delta_{0}\right)$ are SE signals calculated by the new model, and $\tan \left(\Psi_{1}\right)$ and $\cos \left(\Delta_{1}\right)$ are SE signals calculated by the single-film model.

The effective refractive index of resist for the single-film model can be obtained by

$n_{\text {eff }}=\frac{\int n(z) d z}{\int d z}$, and $\quad k_{\text {eff }}=\frac{\int k(z) d z}{\int d z}$

For given Dill parameters, the difference between $n_{\text {unbleached }}$ and $n_{\text {bleached }}$, i.e. $\Delta n=n_{\text {unbleached }}-n_{\text {bleached }}$, as well as the resist thickness will have important impacts on the refractive index distribution. Fig. 2 shows the relative error $\xi$ for $\Delta n=0.02$ and $\lambda=405 \mathrm{~nm}$. When the resist thickness is increased from $0.1 \mu \mathrm{m}$ to $10 \mu \mathrm{m}$, the relative error shows a strong oscillating distribution. However, it tends to increase with higher thickness. Therefore, a single-film model, especially for thick resist characterization, is no longer reliable.

\subsection{Procedure to extract the Dill parameters}

A regression procedure is employed to extract the Dill parameters from the experimentally measured $\tan (\Psi)$ and $\cos (\Delta)$ vs. exposure dose, as shown in Fig. 3. Initial values of the Dill parameters are used as the starting points for simulation of the SE signals. The refractive index of unbleached and bleached resist, the resist thicknesses for the unbleached resist and bleached resist, and the intermediate exposure steps are used as input data to the new simulation program. With these initial parameters, the bleaching data of $\tan (\Psi), \cos (\Delta)$ vs. exposure dose can be calculated. Respectively, the error between calculation and experimental data can be minimized by adjusting the Dill parameters. The optimum values of the Dill parameters can be obtained when the error reaches a limit value. A genetic algorithm is used in the extraction procedure to guarantee that a global minimum error can be reached [11].

\section{Experiment and measurement process}

\subsection{Sample preparation}

Blank 100-mm diameter silicon wafers were prepared applying a clean in a mixture of sulfuric acid $\left(\mathrm{H}_{2} \mathrm{SO}_{4}\right)$ and hydrogen peroxide $\left(\mathrm{H}_{2} \mathrm{O}_{2}\right)(4: 1)$ at $140{ }^{\circ} \mathrm{C}$ followed by a rinse in distilled water and drying

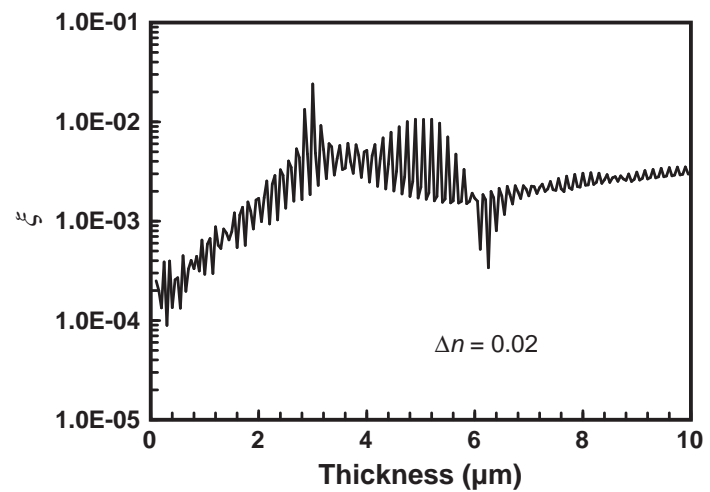

Fig. 2. Difference of simulated SE signals between a single-film resist model and the improved model at $\lambda=405 \mathrm{~nm}$.

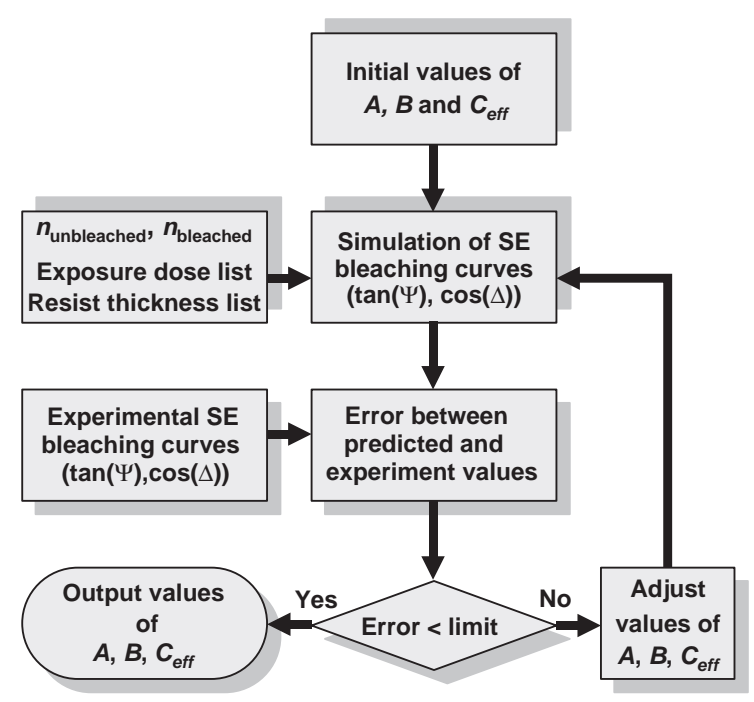

Fig. 3. Computation flow scheme for the calculation of Dill parameters.

in a Semitool PCM328 spin rinse dryer [12]. The wafer was kept in a HMDS (hexamethyldisilazane) environment for a few minutes and then coated with AZ5214E positive-tone photoresist. Resist was deposited manually using a spin coating machine Convac ST146. Spinning speeds were adjusted to $4000 \mathrm{rpm}$ and $1000 \mathrm{rpm}$ to obtain nominal resist thicknesses of $1.5 \mu \mathrm{m}$ and $3.3 \mu \mathrm{m}$, respectively. Prebake was performed on a hotplate in contact mode. Prebake temperature was $90{ }^{\circ} \mathrm{C}$ with a prebake time of $1 \mathrm{~min}$ and $3 \mathrm{~min}$, respectively for $1.5 \mu \mathrm{m}$ and $3.3 \mu \mathrm{m}$ thick resists. Exposure was performed applying the full lamp spectrum without using a mask on a SUSS MicroTec MA6 proximity mask aligner. The exposure dose was adjusted by measuring the light intensity of the Hg-lamp spectrum at $405 \mathrm{~nm}$ ( $h$-line) using a Tamarack 157 UV power meter and, respectively, adjusting the exposure time. The applied nominal exposure doses are summarized in Table 1 together with the prebaking conditions. To determine the exposed doses at $365 \mathrm{~nm}$ and $436 \mathrm{~nm}$, the intensity spectrum of the lamp was integrated at $i-, h$-, and $g$-line peaks and the corresponding intensities were calculated from the known peak areas and the intensity measured at $405 \mathrm{~nm}$.

\subsection{Ellipsometry setup and measurement process}

Spectroscopic ellipsometry measurements were performed on a SOPRA SE-5 ellipsometer in the spectral range of $190 \mathrm{~nm}$ to $1700 \mathrm{~nm}$ with $0.78 \mathrm{~nm}$ steps. To prove the non-invasiveness of the SEmeasurement, 15 consecutive measurements were performed on three different locations on the wafers at an angle of incidence (AOI) of $75^{\circ}$ for the unexposed and the exposed resist with $1.5 \mu \mathrm{m}$ thickness and a nominal dose of $500 \mathrm{~mJ} / \mathrm{cm}^{2}$. On the first site out of three positions, an autofocus procedure was included before each consecutive measurement whereas the measurements on the second and third site were performed without autofocus to identity potential exposure effects by the autofocus process. Measurements for Dill parameter extraction to be further used in the simulation program were performed at two sites on the wafers at angles of incidence of $65^{\circ}, 70^{\circ}$, and $75^{\circ}$ in the spectral range of $190 \mathrm{~nm}$ to $1700 \mathrm{~nm}$ with

Table 1

Parameters for sample preparation.

\begin{tabular}{lll}
\hline $\begin{array}{l}\text { Resist thickness } \\
(\mu \mathrm{m})\end{array}$ & $\begin{array}{l}\text { Prebake time at } 90^{\circ} \mathrm{C} \\
(\mathrm{min})\end{array}$ & $\begin{array}{l}\text { Nominal exposure dose at } 405 \mathrm{~nm} \\
\left(\mathrm{~mJ} / \mathrm{cm}^{2}\right)\end{array}$ \\
\hline 1.5 & 1 & $\begin{array}{l}0,12.5,25,37.5,50,62.5,100,150,200, \\
250,500\end{array}$ \\
3.3 & 3 & $0,25,50,75,100,125,150,250,400,600$ \\
\hline
\end{tabular}


$0.78 \mathrm{~nm}$ steps. For the analysis process, typically, the wavelength range between $200 \mathrm{~nm}$ to $1600 \mathrm{~nm}$ was used. Fig. 4 shows the changes in the $\tan (\Psi)$ and $\cos (\Delta)$ spectra in the wavelength region of $300 \mathrm{~nm}$ to $500 \mathrm{~nm}$ of AZ5214E for a resist thickness of $1.5 \mu \mathrm{m}$ subject to different exposure doses.

\section{Analysis results}

\subsection{Optical constants of resist}

For further analysis of the Dill parameters, the resist thickness of the different samples as well as the refractive index $n$ and the extinction coefficient $k$ at the exposure wavelengths were determined. The film stack was modeled as air as the ambient, a singlelayer resist film and single-crystalline silicon substrate medium, respectively. Tabulated $n, k$ values from the SOPRA database were used to describe the crystalline $\mathrm{Si}$ substrate. The resist can be assumed to be transparent in the visible and near-infrared region, i.e. from $500 \mathrm{~nm}$ to $1600 \mathrm{~nm}$. Resist thickness was extracted in this wavelength regime using a Cauchy model for the refractive index. Regression was performed on $\tan (\Psi)$ and $\cos (\Delta)$ as well as on the normalized Stokes parameters $s_{1}, s_{2}$ calculated thereof [13]. A comparison showed that modeling on $s_{1}, s_{2}$ provided more consistent results probably due to avoiding local minima in the regression of the complex $\tan (\Psi)$ and $\cos (\Delta)$ spectra showing strong oscillations and sharp peaks. From Fig. 4, the increasing absorption of the photoresist and the bleaching effect is clearly visible from the damping of $\tan (\Psi)$ and $\cos (\Delta)$ below $500 \mathrm{~nm}$ which becomes less pronounced at increased doses. Extraction of $n, k$ at the exposure wavelengths was performed by a point-bypoint fit approach over the wavelength region of $300 \mathrm{~nm}$ to $500 \mathrm{~nm}$ to obtain the dispersion curve of resist.
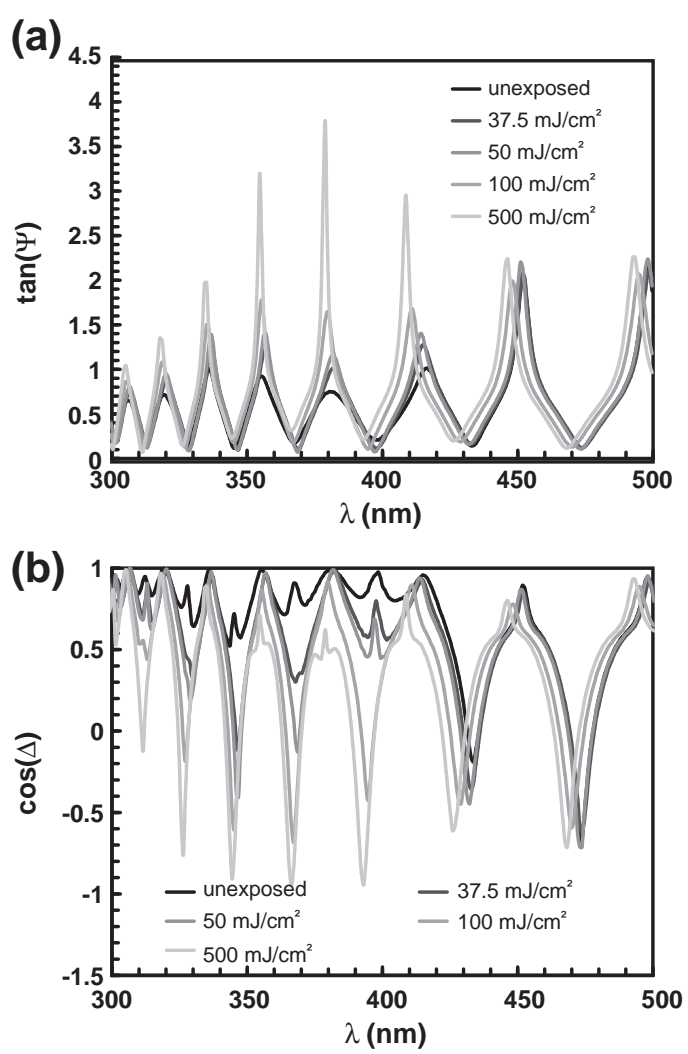

Fig. 4. Changes in the $\tan (\Psi)$ and $\cos (\Delta)$ spectra of AZ5214E with a thickness of $1.5 \mu \mathrm{m}$ subject to different nominal exposure doses in the wavelength region of $300 \mathrm{~nm}$ to $500 \mathrm{~nm}\left(\mathrm{AOI}=75^{\circ}\right)$.
By comparing the $n, k$ values of the 15 repeated measurements on one site, and by comparing the three different site values, it could be shown that no modification of the resist properties is induced by the ellipsometric measurement including the autofocusing process. The refractive indices of a $1.5 \mu \mathrm{m}$ thick resist in the UV range for different exposure doses are shown in Fig. 5. The increased resist absorption below $500 \mathrm{~nm}$ is clearly seen from the increasing $k$ values. It is seen that both $n$ and $k$ values decrease in the UV region with respect to the increase of exposure dose due to the bleaching effect of resist. The refractive index of resist remains constant when the exposure dose is above $500 \mathrm{~mJ} / \mathrm{cm}^{2}$. The refractive indices of unbleached and bleached resists at three exposure wavelengths are shown in Table 2. The obtained $n, k$ values show excellent consistency to the ones provided by the vendor which were obtained at a resist thickness of $1.4 \mu \mathrm{m}$.

\subsection{Extraction results of Dill parameters}

By using the extraction procedure described in Section 2.2, the Dill parameters of resist can be obtained with the experimental bleaching curves of $\tan (\Psi)$ and $\cos (\Delta)$ vs. exposure dose at three incident angles, i.e. $65^{\circ}, 70^{\circ}$, and $75^{\circ}$. The following merit function, $M F$, is used in the regression process due to the improved consistency of results as described in Section 4.1,

$M F=\frac{1}{9 M} \sqrt{\sum_{i=1}^{M} \sum_{j=1}^{3} \sum_{l=1}^{3}\left[\left(s_{1, i, j, l}{ }^{\exp }-s_{1, i, j, l}{ }^{c a l}\right)^{2}+\left(s_{2, i, j, l}{ }^{\exp }-s_{2, i, j, l}{ }^{c a l}\right)^{2}\right]}$

where $s_{1}$ and $s_{2}$ are the normalized Stokes parameters. The indices $i, j$, $l$ represent the indices for the exposure dose steps $i$ given in Table 1 ,
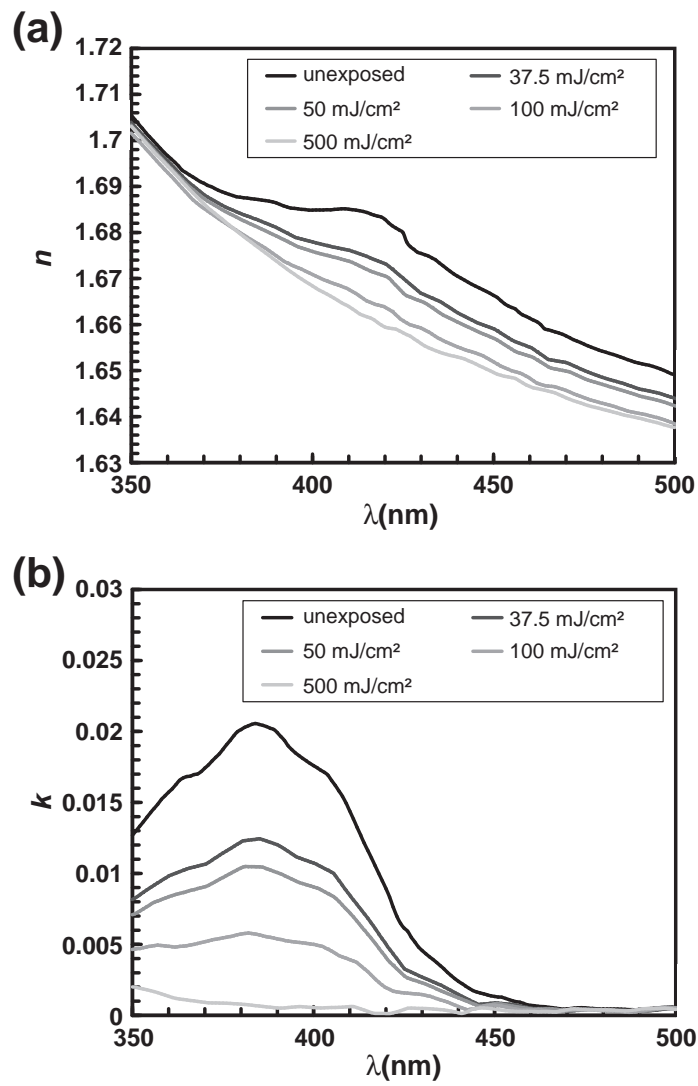

Fig. 5. Measured refractive indices and extinction coefficient of resist with different nominal exposure doses (resist thickness $=1.5 \mu \mathrm{m}$ ). 
Table 2

Experimental values of the refractive index of resist AZ5214E at $365 \mathrm{~nm}, 405 \mathrm{~nm}$ and $436 \mathrm{~nm}$ for a resist thickness of $d=1.5 \mu \mathrm{m}$ and $d=3.3 \mu \mathrm{m}$.

\begin{tabular}{|c|c|c|c|c|}
\hline \multirow[t]{2}{*}{ Resist } & \multicolumn{4}{|l|}{$d=1.5 \mu \mathrm{m}$} \\
\hline & $n_{\text {unexposed }}$ & $k_{\text {unexposed }}$ & $\begin{array}{l}n_{\text {exposed }} \\
\left(500 \mathrm{~mJ} / \mathrm{cm}^{2}\right)\end{array}$ & $\begin{array}{l}k_{\text {exposed }} \\
\left(500 \mathrm{~mJ} / \mathrm{cm}^{2}\right)\end{array}$ \\
\hline $365 \mathrm{~nm}$ & $\begin{array}{l}1.693 \\
\pm 0.001\end{array}$ & $\begin{array}{l}0.017 \\
\pm 0.001\end{array}$ & $\begin{array}{l}1.690 \\
\pm 0.002\end{array}$ & $\begin{array}{l}0.001 \\
\pm 0.001\end{array}$ \\
\hline $405 \mathrm{~nm}$ & $\begin{array}{l}1.685 \\
\pm 0.001\end{array}$ & $\begin{array}{l}0.017 \\
\pm 0.001\end{array}$ & $\begin{array}{l}1.666 \\
\pm 0.002\end{array}$ & $\begin{array}{l}0.001 \\
\pm 0.001\end{array}$ \\
\hline $436 \mathrm{~nm}$ & $\begin{array}{l}1.673 \\
\pm 0.001\end{array}$ & $\begin{array}{l}0.003 \\
\pm 0.001\end{array}$ & $\begin{array}{l}1.654 \\
\pm 0.002\end{array}$ & $<0.001$ \\
\hline \multirow[t]{2}{*}{ Resist } & \multicolumn{4}{|l|}{$d=3.3 \mu \mathrm{m}$} \\
\hline & $n_{\text {unexposed }}$ & $k_{\text {unexposed }}$ & $\begin{array}{l}n_{\text {exposed }} \\
\left(600 \mathrm{~mJ} / \mathrm{cm}^{2}\right)\end{array}$ & $\begin{array}{l}k_{\text {exposed }} \\
\left(600 \mathrm{~mJ} / \mathrm{cm}^{2}\right)\end{array}$ \\
\hline $365 \mathrm{~nm}$ & $\begin{array}{l}1.693 \\
\pm 0.003\end{array}$ & $\begin{array}{l}0.017 \\
\pm 0.001\end{array}$ & $\begin{array}{l}1.693 \\
\pm 0.002\end{array}$ & $\begin{array}{l}0.001 \\
\pm 0.001\end{array}$ \\
\hline $405 \mathrm{~nm}$ & $\begin{array}{l}1.685 \\
\pm 0.003\end{array}$ & $\begin{array}{l}0.017 \\
\pm 0.001\end{array}$ & $\begin{array}{l}1.668 \\
\pm 0.002\end{array}$ & $\begin{array}{l}0.001 \\
\pm 0.001\end{array}$ \\
\hline $436 \mathrm{~nm}$ & $\begin{array}{l}1.673 \\
\pm 0.003\end{array}$ & $\begin{array}{l}0.003 \\
\pm 0.001\end{array}$ & $\begin{array}{l}1.656 \\
\pm 0.001\end{array}$ & $<0.001$ \\
\hline
\end{tabular}

wavelength $(j=1,2,3: 365 \mathrm{~nm}, 405 \mathrm{~nm}, 436 \mathrm{~nm})$ and incident angle values $\left(l=1,2,3: 65^{\circ}, 70^{\circ}, 75^{\circ}\right)$, respectively, and $M$ is the number of exposure doses investigated. Besides the inclusion of the broadband illumination, the change in the resist absorption as well as the variation of the resist refractive index during the exposure process is included in the new model which improves the accuracy of the optical model vs. a single-layer model [8].

The calculation results of the SE signals as shown in Fig. 6 agree very well with the experimental data. The extracted Dill parameters of AZ5214E with thicknesses of $1.5 \mu \mathrm{m}$ and $3.3 \mu \mathrm{m}$ for the three exposure wavelengths are listed in Table 3 . It is seen that the calculated Dill parameters $A$ and $B$ by the new method are in good agreement to the values of $A=0.6181 \mu \mathrm{m}^{-1}$ and $B=0.0314 \mu \mathrm{m}^{-1}$ obtainable for $365 \mathrm{~nm}$ from the vendor. Comparing the Dill parameters extracted at two different thickness values, it can be seen that the Dill $A$ and Dill $B$ parameters show little change when the resist thickness increases from $1.5 \mu \mathrm{m}$ to $3.3 \mu \mathrm{m}$. This means that the absorbance of resist before and after exposure is not dependent on the resist thickness, and the single-layer model is also applicable for the extraction of the $n, k$ values from the ellipsometry data. As discussed in the previous section, $C_{\text {eff }}$ cannot be directly compared to the $C$ parameters obtained from individual exposure at each certain exposure wavelength. These effects are accounted for according to Eq. (6) by calculating the PAC and, therefore, the respective values should be larger. Since the resist has a similar absorption characteristic at $365 \mathrm{~nm}$ and $405 \mathrm{~nm}$, therefore, Dill $C$ parameter should be similar at these two wavelengths. The value of $C_{405 n m \text {,eff }}$ is similar but also larger than the value of $C_{365 \mathrm{~nm}}=0.0284 \mathrm{~cm}^{2} / \mathrm{mJ}$ which is also available from the vendor. However, the Dill parameter $C_{\text {eff }}$ value at $405 \mathrm{~nm}$ is decreasing with increasing thickness. For $365 \mathrm{~nm}$, this decrease in the $C_{365 n m, e f f}$ value cannot be observed. This may be explained by the specific properties of the refractive index value change for the resist at $365 \mathrm{~nm}$ with an exposure dose which is very small (see Fig. 5a). Errors in determination of $C_{365 n m \text {,eff }}$ may be induced by the limited sensitivity of the method whenever the changes in $n$ are small. This may be overcome by increasing the measurement accuracy or modification of the calculation model to update $m(z, t)$ in Eq. (3) whenever the changes in $n$ become too small.

\subsection{Comparison of $n, k$ values between experiment and simulation}

Due to the absorption of light and the standing-wave distribution of the PAC concentration, the refractive index of $n$ and $k$ are not
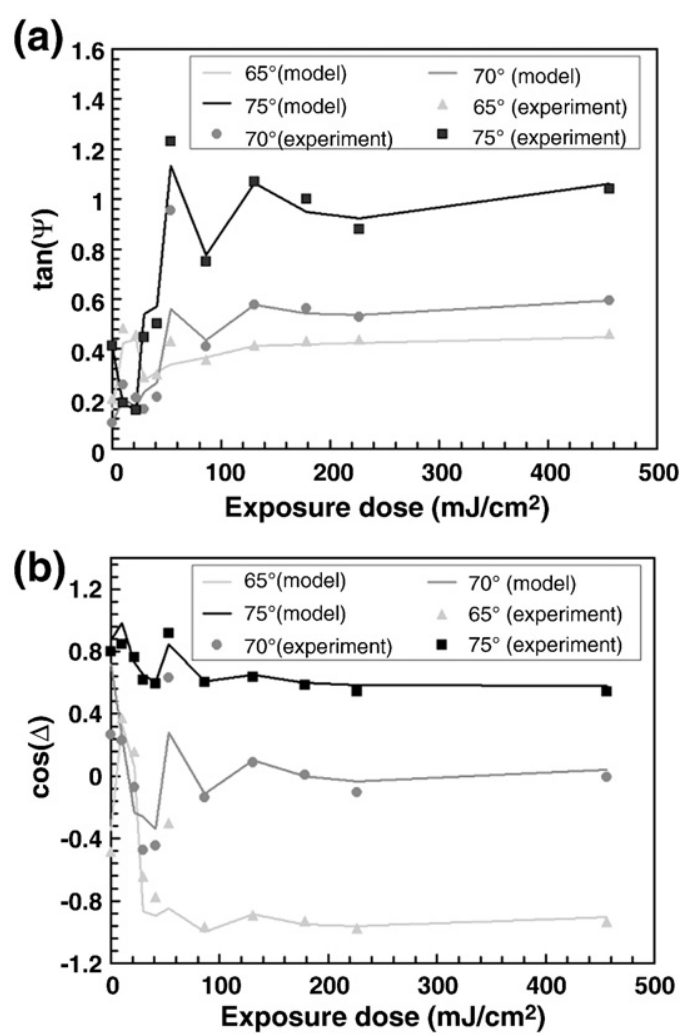

Fig. 6. Comparison of SE signals at $405 \mathrm{~nm}$ between the experiment and calculation results of (a) $\tan (\psi)$ and (b) $\cos (\Delta)$.

uniform in the resist. However, it is very difficult to directly measure the graded refractive index distribution in the resist. Only the unbleached and bleached resists can be treated as uniform layers. One benefit of the method is to use a resist exposure model to simulate the refractive index distribution for intermediate exposure doses. The effective refractive index and extinction coefficient for different exposure doses can be calculated by using Eq. (8). The experimental results obtained by the single-film model and the calculation results obtained by the new model are compared in Fig. 7. It is seen that in most cases, the $n, k$ values obtained with a singlelayer model are very close to the effective $n, k$ values calculated by the new model. Besides differences induced by the different optical models, deviations due to noise in the SE measurement process may occur. Additionally, the correlation between the refractive index $n$ and the resist thickness has to be considered carefully.

Table 3

Comparison of extracted Dill parameters at the resist thicknesses of $1.5 \mu \mathrm{m}$ and $3.3 \mu \mathrm{m}$.

\begin{tabular}{llll}
\hline Resist & $365 \mathrm{~nm}$ & & \\
\cline { 2 - 4 } & $A\left(\mu \mathrm{m}^{-1}\right)$ & $B\left(\mu \mathrm{m}^{-1}\right)$ & $C_{\text {eff }}\left(\mathrm{cm}^{2} \mathrm{~mJ}^{-1}\right)$ \\
\hline$d=1.5 \mu \mathrm{m}$ & 0.600 & 0.028 & 0.014 \\
& \pm 0.006 & \pm 0.002 & \pm 0.001 \\
$d=3.3 \mu \mathrm{m}$ & 0.578 & 0.030 & 0.016 \\
& \pm 0.010 & \pm 0.005 & \pm 0.001 \\
Dill parameters provided by vendor & 0.6181 & 0.0314 & 0.0284 \\
& & & \\
Resist & $405 \mathrm{~nm}$ & & \\
\hline$d=1.5 \mu \mathrm{m}$ & 0.612 & 0.018 & 0.032 \\
& \pm 0.003 & \pm 0.001 & \pm 0.001 \\
$d=3.3 \mu \mathrm{m}$ & 0.629 & 0.019 & 0.018 \\
& \pm 0.010 & \pm 0.001 & \pm 0.001 \\
\hline
\end{tabular}


(a)

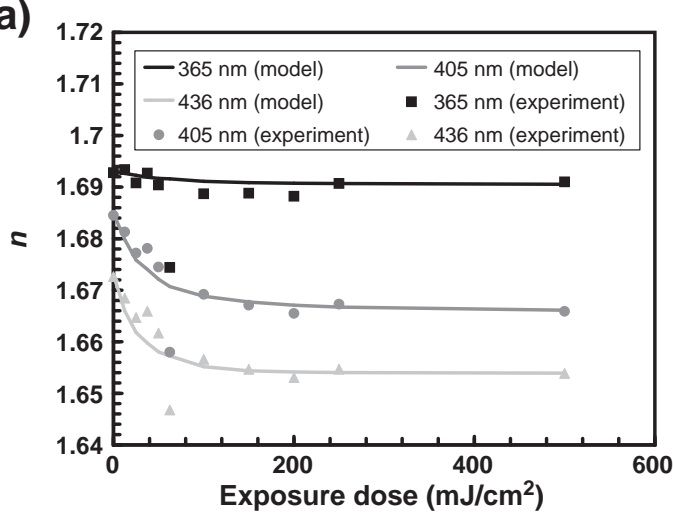

(b)

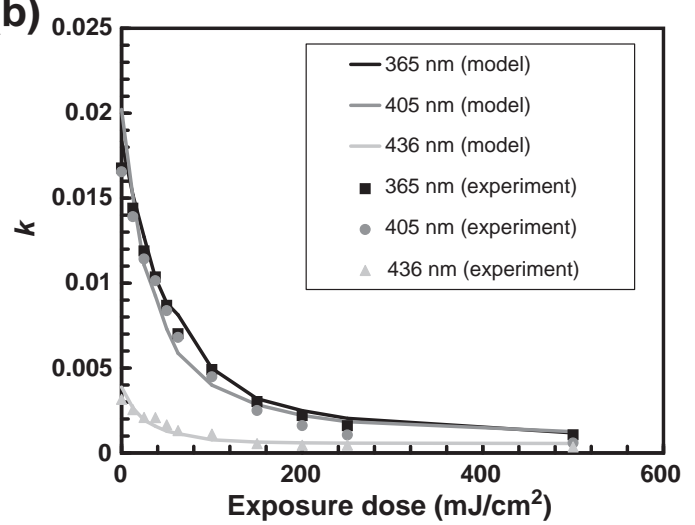

Fig. 7. Comparison of the average refractive index $n$ (a) and the extinction coefficient $k$ (b) between simulation and experimental results for $1.5 \mu \mathrm{m}$ thickness.

\subsection{Effect of Dill parameters variation on image formation in the resist}

Since the Dill parameters are used to characterize the exposure process of resist, their variations will greatly affect the image formation in the resist. Because the Dill $C$ parameter may depend on the resist thickness, the application of a constant Dill $C$ value for different thicknesses will result in simulation errors of the PAC concentration. The PAC concentration for a $3.3 \mu \mathrm{m}$ thick resist after exposure is simulated by the lithography software Dr.LiTHO. A line/ space mask with $10 \mu \mathrm{m}$ pitch and $5 \mu \mathrm{m}$ space is used in the simulation. The total nominal exposure dose is $600 \mathrm{~mJ} / \mathrm{cm}^{2}$. Fig. 8(a) and (b) shows the simulated PAC concentration distributions for the $3.3 \mu \mathrm{m}$ resist with the Dill parameters for $1.5 \mu \mathrm{m}$ and $3.3 \mu \mathrm{m}$ at $405 \mathrm{~nm}$ as shown in Table 3.

It can be seen that the bleaching effect in the resist will only be described correctly if the adapted parameters are used. The resulting PAC concentration will directly affect the calculated development profiles as shown in Fig. 8(c) and hence, derived CD values and focusdose latitude.

\section{Conclusion and future work}

A new extraction technique of Dill parameters based on a rigorous simulation model and SE measurements has been presented. The refractive index $n$ and extinction coefficient $k$ of resist are related with the relative PAC concentration in the resist. With the new method, the Dill parameters can be obtained by directly fitting the bleaching curves of $\tan (\Psi)$ and $\cos (\Delta)$ when single-line exposure is applied. The Dill parameters of AZ5214E resist were extracted for resist thickness of $1.5 \mu \mathrm{m}$ and $3.3 \mu \mathrm{m}$ applying the new method but using broadband illumination. The Dill $A, B$ values are in good agreement with the values provided by the vendor. For broadband
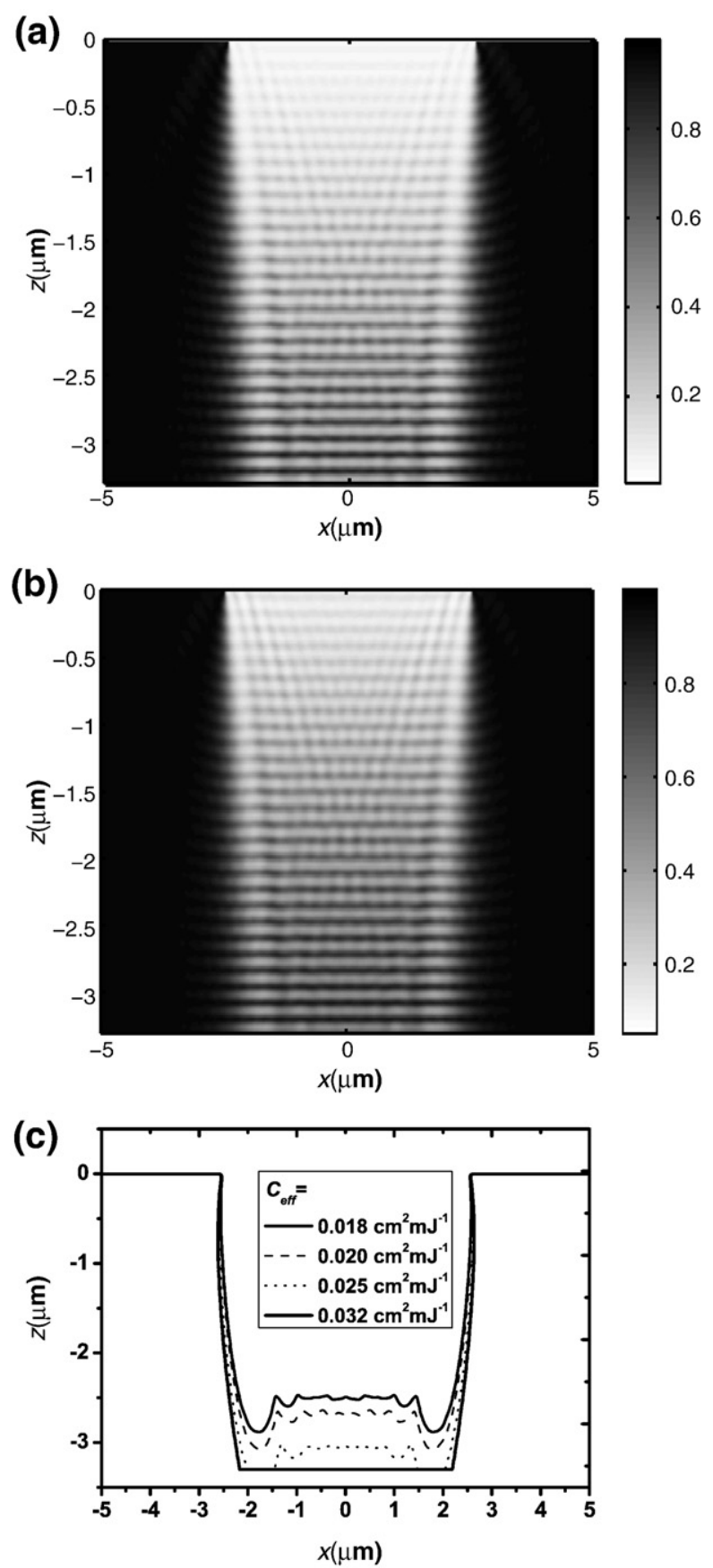

Fig. 8. Simulated PAC concentration distributions (a) and (b) in $3.3 \mu \mathrm{m}$ thick resist: the Dill parameters in (a) and (b) respectively are the values at $1.5 \mu \mathrm{m}$ and $3.3 \mu \mathrm{m}$ for $405 \mathrm{~nm}$ as shown in Table 3, (c) Simulation results of resist profiles with the different Dill parameter $C$.

illumination, an effective Dill $C$ value accounting for the exposure by the different lines was determined. The Dill parameters $A$ and $B$ show little dependence on resist thickness whereas the Dill $C$ value will depend on the resist thickness due to the resist bleaching. Limitations on the accuracy of the Dill $C$ value determination were discussed. With the determined Dill parameters, the influence of the parameters determined at different resist thickness on PAC concentration and resist profile was demonstrated.

As it could be shown that the new analysis method will provide means for exact determination of the Dill parameters, experimental work will be continued for resist thickness above $10 \mu \mathrm{m}$. Here, the advantage can be taken, that merely $n, k$ values for the unexposed and 
fully exposed resist need to be determined. The sensitivity of the method is determined by the difference of refractive index $n$ between the unexposed and fully exposed resists. This may be improved by increasing the measurement accuracy of the refractive index $n$ or modification of the calculation model whenever the changes in $n$ before and after full exposure become too small. Additionally, a monochromatic exposure source should be applied for determination of the individual Dill $C$ parameters.

\section{Acknowledgement}

We highly acknowledge the preparation of the resist samples by Ms. Stephanie Natzer.

\section{References}

[1] C. Liu, Adv. Mater. 19 (2007) 3783.

[2] F.H. Dill, IEEE Trans. Electron Devices 22 (1975) 440.
[3] S. Liu, J. Du, X. Duan, B. Luo, X. Tang, Y. Guo, Z. Cui, C. Du, J. Yao, Microelectron. Eng. 78-79 (2005) 490.

[4] C.A. Mack, J. Electrochem. Soc. 134 (1987) 148.

[5] G.G. Arthur, B. Martin, in: C.A. Mack, T. Stevenson (Eds.), Lithography for Semiconductor Manufacturing II, Proc. SPIE-Int. Soc. Opt. Eng., 4404, 2001, p. 209.

[6] C.L. Henderson, S. Pancholi, S.A. Chowdhury, C.G. Willson, R.R. Dammel, in: R.G. Tarascon-Auriol (Ed.), Advances in Resist Technology and Processing XIV, Proc. SPIE-Int. Soc. Opt. Eng., 3049, 1997, p. 816.

[7] P. Schiavone, S. Bach, in: W. Conley (Ed.), Advances in Resist Technology and Processing XV, Proc. SPIE-Int. Soc. Opt. Eng., 3333, 1998, p. 1251.

[8] P. Boher, C. Defranoux, J.P. Piel, J.L. Stehle, in: W. Conley (Ed.), Advances in Resist Technology and Processing XVI, Proc. SPIE-Int. Soc. Opt. Eng. 3678 Microlithography, 1999, p. 126.

[9] C.L. Henderson, C.G. Willson, R.R. Dammel, R.A. Synowicki, in: R.G. TarasconAuriol (Ed.), Advances in Resist Technology and Processing XIV, Proc. SPIE-Int. Soc. Opt. Eng., 3049, 1997, p. 585.

[10] T. Fuhner, T. Schnattinger, G. Ardelean, A. Erdmann, in: D.G. Flagello (Ed.), Optica Microlithography XX, Proc. SPIE-Int. Soc. Opt. Eng., 6520, 2007, p. 65203F

[11] T. Fuhner, A. Erdmann, S. Seifert, J. Micro Nanolith. MEMS MOEMS 6 (2007) 031006.

[12] W. Kern, J. Electrochem. Soc. 137 (1990) 1887.

[13] H. Fujiwara, Spectroscopic Ellipsometry: Principles and Applications, John Wiley \& Sons, Chichester, UK, 2007, p. 75. 